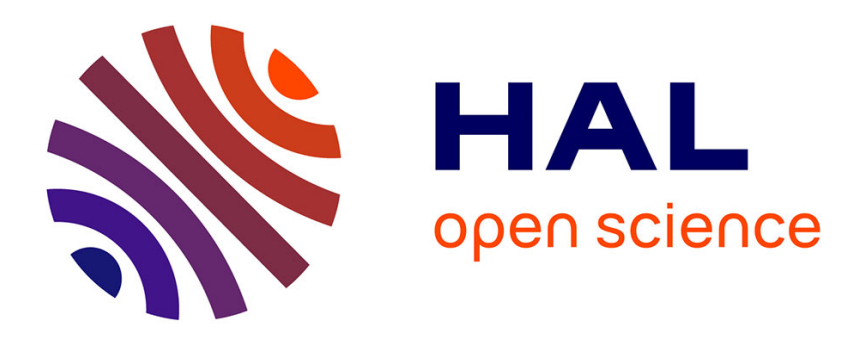

\title{
Transition near the edge of a rotating disk
}

Benoît Pier

\section{To cite this version:}

Benoît Pier. Transition near the edge of a rotating disk. Journal of Fluid Mechanics, 2013, 737, pp.R1. 10.1017/jfm.2013.578 . hal-00907846

\section{HAL Id: hal-00907846 https://hal.science/hal-00907846}

Submitted on 21 Nov 2013

HAL is a multi-disciplinary open access archive for the deposit and dissemination of scientific research documents, whether they are published or not. The documents may come from teaching and research institutions in France or abroad, or from public or private research centers.
L'archive ouverte pluridisciplinaire HAL, est destinée au dépôt et à la diffusion de documents scientifiques de niveau recherche, publiés ou non, émanant des établissements d'enseignement et de recherche français ou étrangers, des laboratoires publics ou privés. 


\section{JFM RAPIDS journals.cambridge.org/rapids}

\section{Transition near the edge of a rotating disk}

\section{Benoît Pier $\dagger$}

Laboratoire de mécanique des fluides et d'acoustique, École centrale de Lyon - CNRS - Université de Lyon 1 - INSA Lyon, 36 avenue Guy-de-Collongue, 69134 Écully CEDEX, France

(Received 4 October 2013; revised 23 October 2013; accepted 28 October 2013)

The rotating-disk boundary layer is generally considered as an example of a flow that displays a robust transition from laminar to turbulent régimes. By taking into account disks of finite radius, Healey (J. Fluid Mech., vol. 663, 2010, pp. 148-159) has predicted a stabilizing effect of the boundary condition, but Imayama et al. (J. Fluid Mech., vol. 716, 2013, pp. 638-657) were unable to confirm this prediction experimentally. Following these contradictory results, the present experimental investigation revisits the rotating-disk boundary layer, without any artificially imposed excitation, and studies in further detail the dynamics prevailing in the region closely surrounding the edge of the disk, as well as the flow beyond the disk. Azimuthal mean velocities and fluctuation amplitudes are recorded with small steps in radial and axial directions for a wide range of disk sizes. An objective criterion is used to define the onset of fluctuations consistently over a large data set. Two distinct mechanisms for the onset of fluctuations are identified. In particular, it is found that the flow over the edge of the disk acts as a strong source of fluctuations. Explanations and suggestions for a possible reconciliation of previous studies are given.

Key words: boundary layer stability, instability, transition to turbulence

\section{Context}

Ever since the pioneering work of von Kármán (1921), the boundary layer due to a disk rotating in otherwise still fluid has served as the archetypical three-dimensional boundary layer (Reed \& Saric 1989; Saric, Reed \& White 2003; Launder, Poncet \& Serre 2010). This flow is known for its robust laminar-turbulent transition occurring at a radial position closely corresponding to the onset of local absolute instability (Lingwood 1995, 1996).

Assuming a disk of infinite extent, previous studies have established the global linear stability of the base boundary-layer flow (Garrett 2002; Davies \& Carpenter

$†$ Email address for correspondence: benoit.pier@ec-lyon.fr 


\section{B. Pier}
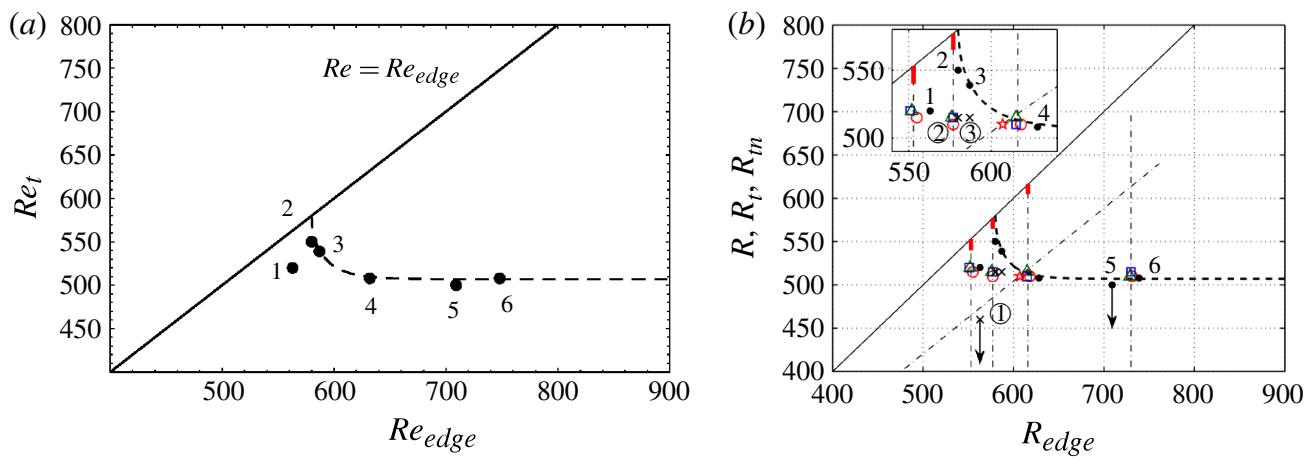

FIGURE 1. Dependence of transition radius $\left(R e_{t}\right.$ or $\left.R_{t}\right)$ on the size of the disk $\left(R e_{\text {edge }}\right.$ or $\left.R_{\text {edge }}\right)$ : (a) reproduced from Healey (2010), figure 7(a); (b) reproduced from Imayama et al. (2013), figure 8 . The numbers 1-6 refer to transition data from different experiments used in both papers.

2003), while the nonlinear behaviour can be explained by a scenario involving both local primary and secondary absolute instabilities (Pier 2003). Local absolute instability is only a necessary but not a sufficient condition for global linear instability (Huerre \& Monkewitz 1990). In contrast, nonlinear global modes (aka 'elephant' global modes (Pier \& Huerre 2001)) are triggered by a sharp front at the transition from local convective to absolute instability; thus, the existence of local absolute instability is a necessary and sufficient condition for global nonlinear instability (Pier, Huerre \& Chomaz 2001). It turns out that the rotating-disk flow precisely falls into the category of linearly stable but nonlinearly unstable systems.

By considering spatially varying systems of finite extent, a recent theoretical study by Healey (2010) has shown that the presence of a downstream boundary condition may have a destabilizing effect on the base state and a stabilizing effect on the nonlinear state. Using a simple nonlinear model, Healey (2010) has shown that the front which appears at the onset of absolute instability when the boundary is far from the front, moves slightly downstream when the boundary approaches the front. For the rotating-disk configuration, the transition radius is thus expected to increase when the size of the disk is reduced. This prediction may be supported by plotting experimentally observed transition radii found in the literature; see figure 7(a) of Healey (2010), reproduced here as figure 1(a), where the numbers 1-6 refer to transition data from different experiments. However, this theory is unable to quantitatively assess this stabilizing effect for the rotating disk since the nonlinear interaction terms are difficult to quantify for this flow.

Following these theoretical predictions, the edge effects on rotating-disk transition have been experimentally studied by Imayama, Alfredsson \& Lingwood (2013). Three different edge conditions and a range of edge Reynolds numbers have been investigated. The authors concluded that no obvious variation in the transition location due to the proximity to the edge of the disk had been observed in the study; see figure 8 of Imayama et al. (2013), reproduced here as figure 1(b). Note, however, that data measured within 10 boundary-layer units from the edge were removed in that study since the mean flow was seen to deviate from the Kármán similarity solution in this region, and that the values of the transition radii already used by Healey (2010) were reinterpreted in Imayama et al. (2013), using different definitions of onset. 


\section{Transition near the edge of a rotating disk}

In view of these contradictory results, there is clearly a need for new measurements covering a wide range of disk radii and for an unbiased criterion to define the transition location consistently over a large data set. From a long history of rotatingdisk experiments (Theodorsen \& Regier 1944; Smith 1947; Gregory, Stuart \& Walker 1955; Chin \& Litt 1972; Fedorov et al. 1976; Kobayashi, Kohama \& Takamadate 1980; Malik, Wilkinson \& Orszag 1981; Wilkinson \& Malik 1985; Lingwood 1996; Othman \& Corke 2006; Imayama, Alfredsson \& Lingwood 2012; Imayama et al. 2013; Siddiqui et al. 2013), the reported radii for onset of transition display significant scatter. This scatter may be attributable to edge effects, but also to the use of different definitions of onset radius. Although the rotating-disk boundary layer is usually associated with a sharp laminar-turbulent transition, detailed measurements by Siddiqui et al. (2013) have shown that there exists a significant intermediate nonlinear régime, and that the extent of this intermediate region (of the order of 50 units) also depends on disk-normal distance. This observation again calls for new measurements and for the use of a consistent criterion.

Therefore the present investigation aims to make a contribution to our understanding of the dynamics prevailing near the edge, as well as the flow behaviour beyond the disk. By considering a wide range of non-dimensional disk sizes, the aim is to perform measurements with small steps in radial and normal directions and to use an objective criterion to gain further insight into the role played by the edge region in the global dynamics.

\section{Experimental setup and procedure}

The experimental facility (see figure 2) used in the present investigation has been improved following Siddiqui (2011)'s thesis and consists of a synthetic resin disk of $R_{e}^{\star}=250 \mathrm{~mm}$ radius that is rotated at constant angular velocity, up to 2000 r.p.m. The disk surface topography was characterized using by a linear variable differential transducer (LVDT) and adjustments were made so as to achieve best alignment with a plane normal to the rotation axis. The residual out-of-flatness was less than $20 \mu \mathrm{m}$ (i.e. the entire disk surface is within two parallel planes that are no more than $20 \mu \mathrm{m}$ apart) and the azimuthal imbalance is less than $10 \mu \mathrm{m}$ (i.e. below any fixed point in the laboratory frame, the surface of the disk travels by less than $10 \mu \mathrm{m}$ under rotation). The edge of the disk is a sharp right angle (see figure $2 b$ ), and the disk protrudes $23 \mathrm{~mm}$ above the aluminium plate that holds it. Since a typical boundarylayer thickness is less than $0.5 \mathrm{~mm}$, it is thus reasonable to assume that this setup is a good approximation of a disk of finite radius and infinite thickness.

Following Siddiqui (2011), local velocity measurements are carried out via constanttemperature hot-wire anemometry, which is particularly suitable for measuring flows with fast fluctuations at fixed points in space. A single Dantec hot wire of type 55P01 is used, positioned parallel to the disk surface and aligned in the radial direction so as to measure the azimuthal flow component. A high-precision computer-controlled traversing mechanism is used for positioning the probe in the radial and axial directions with precisions of $20 \mu \mathrm{m}$ and $2 \mu \mathrm{m}$ respectively. The accessible range of radial positions is such that measurements up to $20 \mathrm{~mm}$ beyond the edge of the disk are possible. In the axial direction, the hot wire can reach down to $9 \mathrm{~mm}$ below the disk surface. Due to the size of the hot-wire probe $(5 \mathrm{~mm})$, it is safe to measure below the disk surface only for $R^{\star} \geqslant 253 \mathrm{~mm}$.

Here, the constant boundary-layer thickness is proportional to $\delta=\sqrt{v / \Omega}$, where $v$ is the kinematic viscosity and $\Omega$ the disk rotation rate. Since all distances are non- 
(a)

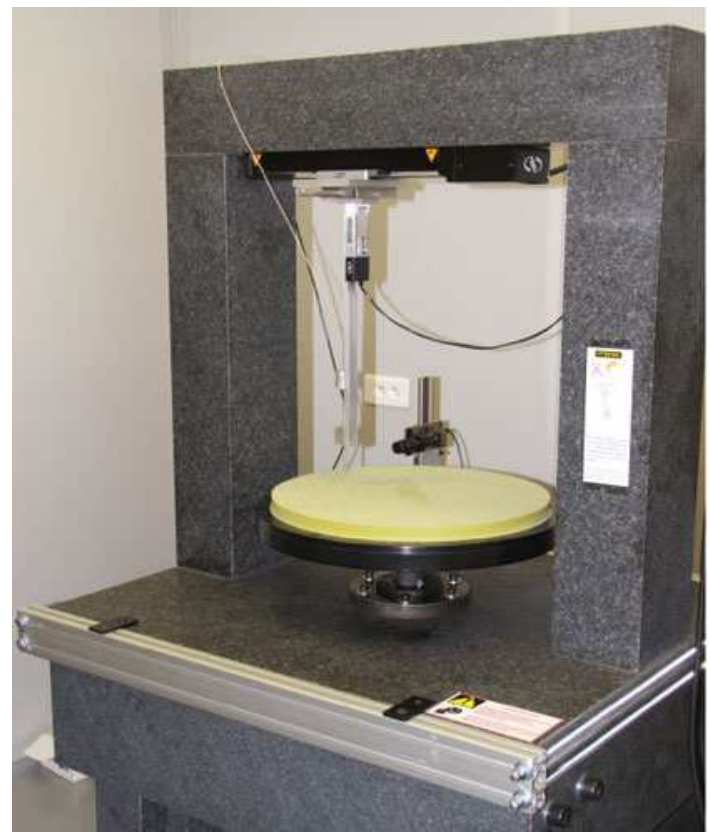

(b)

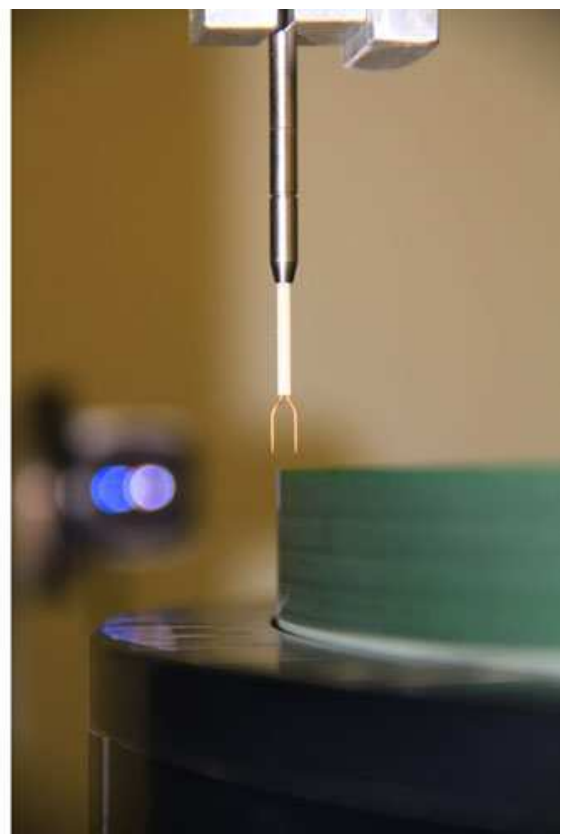

FIGURE 2. Experimental setup. (a) General view of disk and two-axes traversing mechanism for computer-controlled measurements. (b) Hot-wire probe above the sharp edge of the disk, protruding $23 \mathrm{~mm}$ out of the aluminium plate.

dimensionalized by $\delta$, the non-dimensional disk-edge radius $R_{e}=R_{e}^{\star} / \delta$ may be varied by adjusting the disk rotation rate. Then, velocity measurements are automatically performed over specified ranges of non-dimensional radial and axial positions, $R$ and $Z$; at each position, data are typically acquired over 100 disk revolutions. Velocities are always non-dimensionalized by the local disk velocity: $V=V^{\star} /(R \delta \Omega)$.

\section{Results}

Mean azimuthal velocity profiles are shown in figure 3 for $R_{e}=400,500,550$ and 600. Symbols correspond to measurements, while the solid curve indicates the von Kármán similarity solution. These plots show that the azimuthal velocities depart from the Kármán profile either when transition starts $(R \gtrsim 500)$ or when the edge is approached $\left(R \gtrsim R_{e}\right)$. Strong azimuthal shear prevails in the boundary layer over the entire disk $\left(R<R_{e}\right.$ and $\left.0<Z<4\right)$. Beyond the disk edge $\left(R>R_{e}\right)$, the azimuthal shear rapidly decays and the velocity profiles flatten out over the entire $Z$-range, above and below the disk surface. Even when the flow is expected to remain laminar up to the edge of the disk (e.g. $R_{e}=400$, figure $3 a$ ), the presence of the boundary is felt about 10 boundary-layer units inboard. For this reason, Imayama et al. (2013) removed all data measured close to the outer edge from their results and discussions. While Healey (2010)'s theory assumes a point-like boundary condition and vanishing fluctuations at this point, the cross-over from the boundary layer prevailing over the 

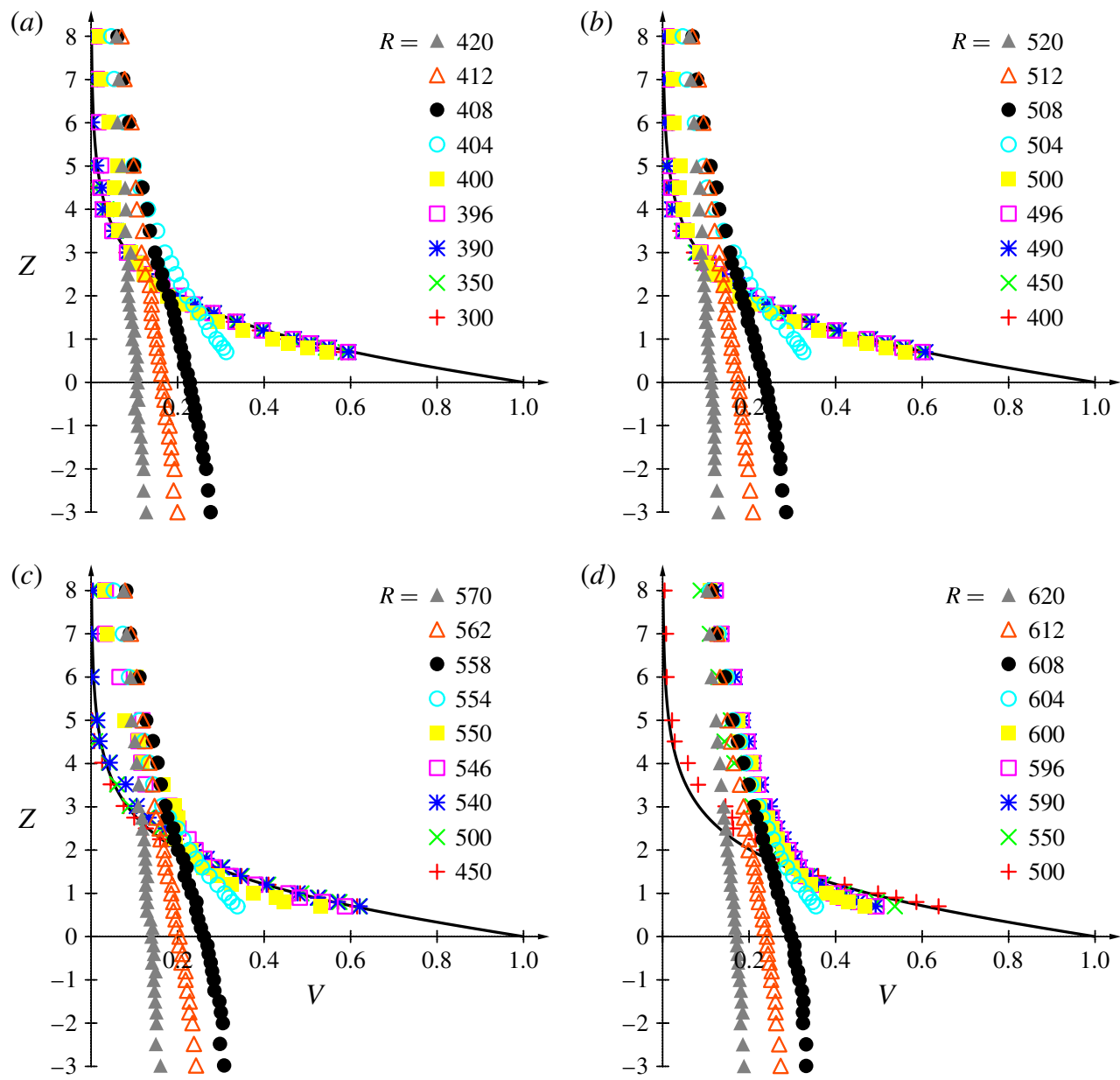

FIGURE 3. Mean azimuthal velocity profiles obtained with (a) $R_{e}=400,(b) R_{e}=500$, (c) $R_{e}=550,(d) R_{e}=600$. The solid curve indicates the Kármán similarity profile and symbols correspond to measurements at the specified non-dimensional radial positions.

disk surface to the low-velocity region beyond the disk clearly occurs in a more gradual way. We believe therefore that an investigation of the edge effects should precisely take into account this cross-over region.

The structure of this cross-over region is more clearly illustrated in figure 4 , where radial sections of the mean azimuthal velocity are plotted for different edge radii and different normal distances. For $R<R_{e}$ and $R<500$ all plots are almost horizontal lines, which shows that they closely follow the Kármán similarity solution, whatever the disk-normal distance $Z$. Beyond the edge, $R>R_{e}$, the velocities depend much less on $Z$ and rapidly drop with increasing radial distance. It is only for larger edge radii $\left(R_{e}>550\right)$ that a significant departure from the Kármán solution occurs before the disk edge is reached.

The amplitude of the fluctuations around the basic flow has been characterized by $V_{r m s}$, the root-mean-square (r.m.s.) value of the velocity. Figure 5 shows the radial 


\section{B. Pier}

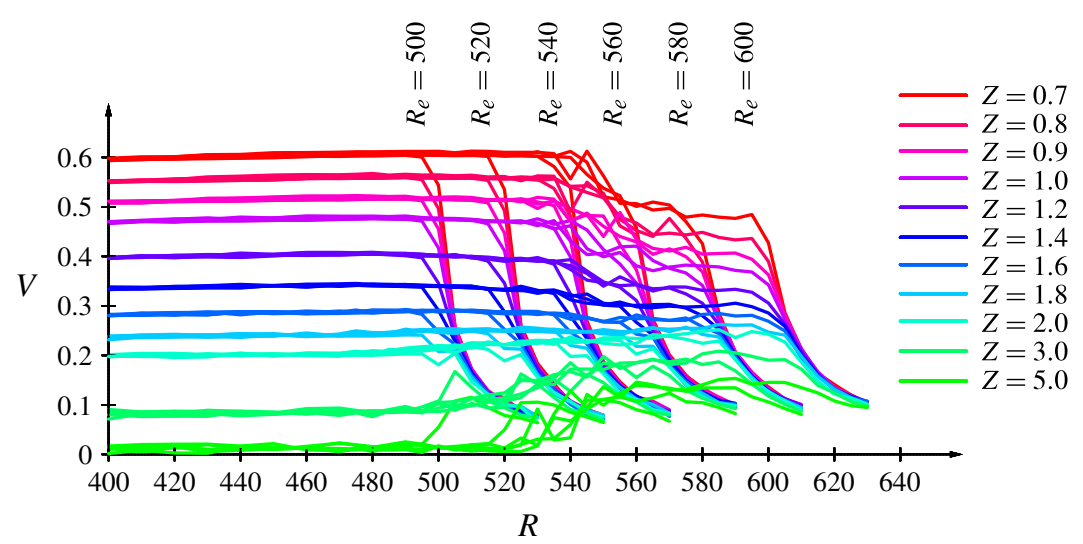

FIGURE 4. Radial traverses of mean azimuthal velocity for a range of disk-normal distances $Z$ and edge radii $R_{e}$.
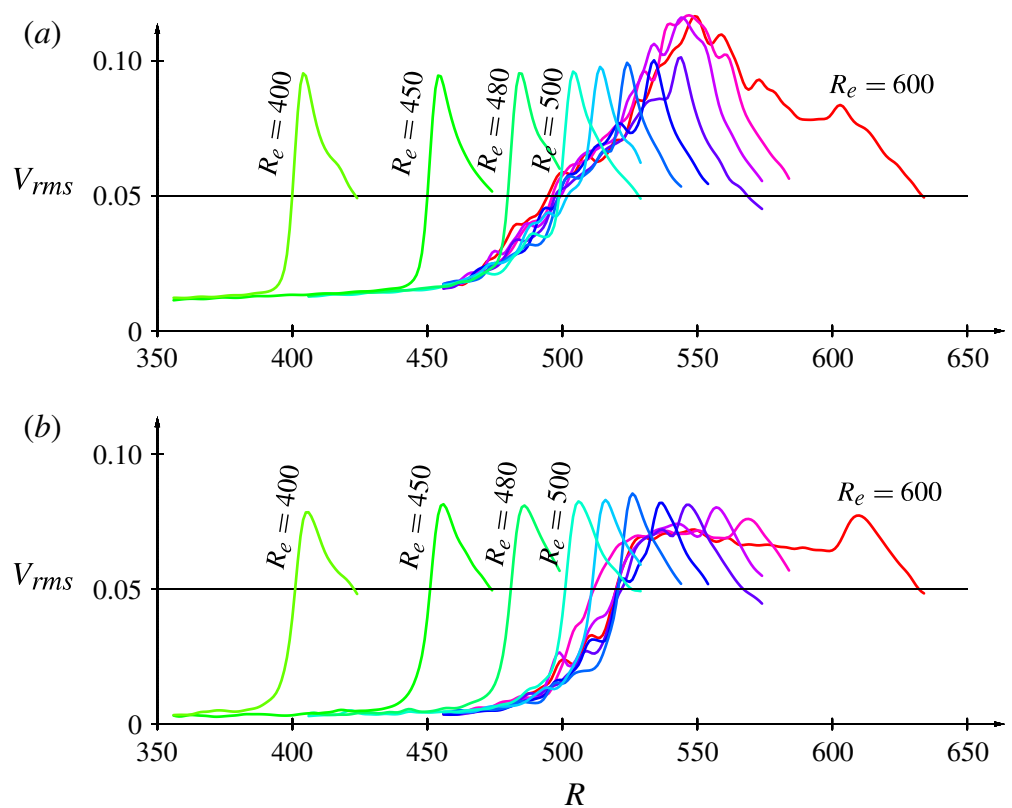

FIGURE 5. Radial evolution of $V_{r m s}$ at $Z=1(a)$ and $Z=3(b)$ for $R_{e}=400,450,480,500,510$, $520,530,540,550,560$ and 600.

evolution of the fluctuating amplitude for a range of $R_{e}$, measured at $Z=1$ (figure $5 a$ ) and $Z=3$ (figure $5 b$ ). These plots show two distinct features. For $R_{e} \lesssim 500$, the boundary layer remains unperturbed over most of the disk surface and the r.m.s. values rapidly increase near the edge of the disk to reach a maximum value near $R_{e}+5$ beyond which they decay again. For $R_{e} \gtrsim 500$, fluctuations start to rise as $R=500$ is approached and continue for the rest of the boundary layer.

These measurements clearly indicate that the near-edge region acts as a strong source of noise, even at very low $R_{e}$ (i.e. at very low disk rotation rates). At a given disk-normal distance $Z$, this sudden increase of fluctuations in the vicinity of 


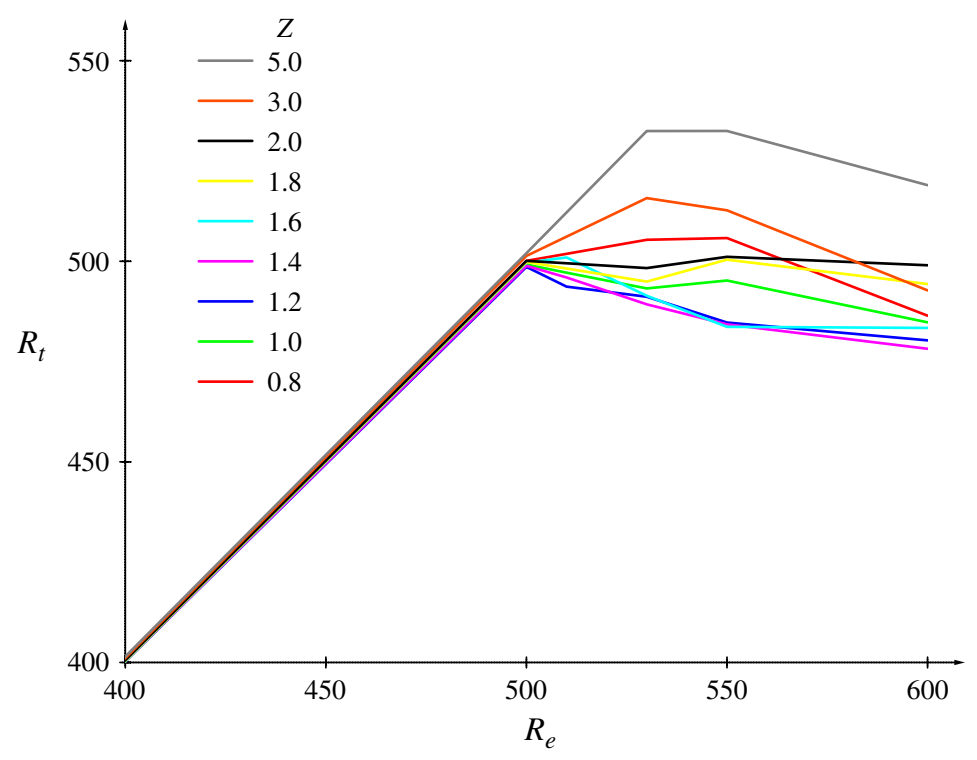

FIGURE 6. Dependence of onset radius $R_{t}$ on edge location $R_{e}$ for different values of disk-normal distance $Z$.

the edge has a characteristic shape that hardly depends on the value of $R_{e}$, provided that $R_{e}<500$. For larger disks, $R_{e}>500$, a slow increase of fluctuation amplitude is observed to start around $R=450$ and to saturate around $R=550$. This more gradual development of fluctuation amplitude does not seem to be much affected by the value of the edge radius. For much larger disks (see plots corresponding to $R_{e}=600$ in figure 5), large-amplitude fluctuations are fully developed before the edge is reached, and the r.m.s. values display only a weak peak at the edge before decaying beyond the edge.

In order to characterize more precisely the influence of the edge, an objective criterion for the onset of finite-amplitude fluctuations is required. Here we define $R_{t}$ as the radial position where the above r.m.s. values cross the value 0.05 (thin horizontal lines in figure 5). Note that, due to the steep slope of the curves in figure 5, this criterion yields onset values that are largely insensitive to the precise threshold value used (here 0.05). Applying this criterion to the data acquired over a large number of experimental runs yields the dependence of the onset radius $R_{t}$ on the edge location $R_{e}$ and disk-normal distance $Z$, shown in figure 6. These plots show that for all $R_{e} \leqslant 500$ and $Z \leqslant 5$, onset always occurs at the disk edge: $R_{t}=R_{e}$. For $R_{e}>500$, the onset radius $R_{t}$ no longer linearly increases with $R_{e}$ and eventually reaches a nearly constant value. The onset radius moreover significantly depends on the disk-normal distance $Z$ : earliest onset is observed for $Z \simeq 1.4$, while it is delayed in the regions further above the disk. Since for most of these curves the onset radius $R_{t}$ slightly decreases with increasing $R_{e}$ for $R_{e}>500$, this could be interpreted as pointing towards a weakly stabilizing edge effect, as predicted by Healey (2010). However, considering only curves derived from measurements at $Z=2.0$ and $Z=1.8$, which are nearly flat, one could also argue that the stabilizing edge effect is negligible at these disk-normal distances. 


\section{B. Pier}

\section{Summary and discussion}

In the present investigation, the near-edge region of the rotating-disk boundary layer has been characterized in detail for a wide range of non-dimensional disk radii. Mean azimuthal velocity measurements reveal how the cross-over from the boundary layer prevailing over the disk surface to the low-velocity region beyond the disk occurs.

Detailed measurements of the spatial development of fluctuating amplitudes have been carried out, using small steps in $R$ and $Z$ for a range of disk radii $R_{e}$. The onset radius $R_{t}$ has then been obtained via automatic data processing, using the same criterion consistently across a large data set. It has been found that for small disks, i.e. $R_{e}<500$, onset always occurs at the disk edge: $R_{t}=R_{e}$ independently of $Z$. For large disks, i.e. $R_{e}>500$, it is observed that the onset radius $R_{t}$ depends weakly on $R_{e}$ and also significantly on $Z$, and no stabilizing trend can be clearly established.

In view of these results, it seems that Healey (2010)'s theory cannot be confirmed and also that any attempt to compare data obtained via different experiments and criteria cannot be justified. Indeed, the present investigation has shown that the scatter of $R_{t}$ due to different values of $Z$ is of the same order as the data given by Healey (2010) or Imayama et al. (2013) (see figure 1).

Two distinct mechanisms have been identified for the onset of fluctuations:

Larger disks (typically $R_{e}>500$ ) display a gradual increase of fluctuating amplitude for $450<R<550$, which is mostly independent of disk size. This may be interpreted as a self-sustained boundary-layer global mode (Pier 2003), triggered by absolute instability near $R=500$ and largely insensitive to edge effects.

Smaller disks (typically $R_{e}<500$ ) display a sharp increase of fluctuating amplitude in the vicinity of the edge. This shows that, even at low rotation rates, the flow over the edge of the disk is strongly unstable. The flow over the edge is a combination of a radial wall jet (shooting over the edge) and an azimuthal shear layer that rapidly decay with radial distance. We conjecture that this flow over the edge is locally absolutely unstable, thus triggering another self-sustained global mode that cannot be suppressed.

By masking any stabilization due to the zero-fluctuation outlet condition, this strong source of fluctuations could provide an explanation for the inapplicability of the theory (Healey 2010). A possible reconciliation between Healey (2010)'s theory and Imayama et al. (2013)'s interpretation might be achieved if the theory could be modified so as to model the downstream boundary condition as a source of random noise rather than by vanishing fluctuating amplitude.

Further theoretical investigations are planned to obtain the base flow solutions prevailing beyond the disk and their stability characteristics, in order to study the possible existence of a self-sustained edge global mode and its competition with the boundary-layer global mode.

\section{Acknowledgements}

The author thanks J. J. Healey, S. Imayama, P. H. Alfredsson and R. J. Lingwood for stimulating discussions and for convincing him to undertake the present work. Invaluable technical assistance by A. Azouzi, H. Correia, R. Michelet, C. Nicot and B. Poirel is gratefully acknowledged. This research was partially supported by Agence nationale de la recherche (Project 'Microsillon'). 


\section{References}

Chin, D.-T. \& LiTt, M. 1972 An electrochemical study of flow instability on a rotating disk. J. Fluid Mech. 54, 613-625.

DAvies, C. \& CARPenter, P. W. 2003 Global behaviour corresponding to the absolute instability of the rotating-disk boundary layer. J. Fluid Mech. 486, 287-329.

Fedorov, B. I., Plavnik, G. Z., Prokhorov, I. V. \& Zhukhovitskit, L. G. 1976 Transitional flow conditions on a rotating disk. J. Engng Phys. 31, 1448-1453.

GARrett, S. J. 2002 The stability and transition of the boundary layer on rotating bodies. PhD thesis, University of Cambridge.

Gregory, N., Stuart, J. T. \& WALKer, W. S. 1955 On the stability of three-dimensional boundary layers with application to the flow due to a rotating disk. Phil. Trans. R. Soc. Lond. A 248, 155-199.

HeAley, J. J. 2010 Model for unstable global modes in the rotating-disk boundary layer. J. Fluid Mech. 663, 148-159.

Huerre, P. \& Monkewitz, P. A. 1990 Local and global instabilities in spatially developing flows. Annu. Rev. Fluid Mech. 22, 473-537.

Imayama, S., Alfredsson, P. H. \& Lingwood, R. J. 2012 A new way to describe the transition characteristics of a rotating-disk boundary-layer flow. Phys. Fluids 24, 031701.

Imayama, S., Alfredsson, P. H. \& Lingwood, R. J. 2013 An experimental study of edge effects on rotating-disk transition. J. Fluid Mech. 716, 638-657.

VON KÁRmÁn, Th. 1921 Über laminare und turbulente Reibung. Z. Angew. Math. Mech. 1, 233-252.

Kobayashi, R., Kohama, Y. \& Takamadate, C. 1980 Spiral vortices in boundary layer transition regime on a rotating disk. Acta Mechanica 35, 71-82.

Launder, B., Poncet, S. \& Serre, E. 2010 Laminar, transitional, and turbulent flows in rotor-stator cavities. Annu. Rev. Fluid Mech. 42, 229-248.

LINGWOOD, R. J. 1995 Absolute instability of the boundary layer on a rotating disk. J. Fluid Mech. 299, 17-33.

LiNGWOOD, R. J. 1996 An experimental study of absolute instability of the rotating-disk boundary-layer flow. J. Fluid Mech. 314, 373-405.

Malik, M. R., Wilkinson, S. P. \& OrSZAG, S. A. 1981 Instability and transition in rotating disk flow. AIAA J. 19, 1131-1138.

OTHMAN, H. \& CORKE, T. 2006 Experimental investigation of absolute instability of a rotating-disk boundary layer. J. Fluid Mech. 565, 63-94.

PIER, B. 2003 Finite-amplitude crossflow vortices, secondary instability and transition in the rotating-disk boundary layer. J. Fluid Mech. 487, 315-343.

Pier, B. \& Huerre, P. 2001 Nonlinear synchronization in open flows. J. Fluids Struct. 15, 471-480.

Pier, B., Huerre, P. \& Chomaz, J.-M. 2001 Bifurcation to fully nonlinear synchronized structures in slowly varying media. Physica D 148, 49-96.

REeD, H. L. \& SARIC, W. S. 1989 Stability of three-dimensional boundary layers. Annu. Rev. Fluid Mech. 21, 235-284.

SARIC, W. S., Reed, H. L. \& White, E. B. 2003 Stability and transition of three-dimensional boundary layers. Annu. Rev. Fluid Mech. 35, 413-440.

SIDDIQUi, M. E. 2011 Experimental study of natural and forced instabilities and transition of a rotating-disk boundary-layer flow. PhD thesis, École centrale de Lyon, Écully, France.

Siddiqui, M. E., Mukund, V., Scott, J. \& Pier, B. 2013 Experimental characterization of transition region in rotating-disk boundary layer. Phys. Fluids 25, 034102.

SMITH, N. H. 1947 Exploratory investigation of laminar-boundary-layer oscillations in a rotating disk. NACA Tech. Rep. TN 1227.

Theodorsen, T. \& Regier, A. 1944 Experiments on drag of revolving disks, cylinders, and streamline rods at high speeds. NACA Tech. Rep. 793.

Wilkinson, S. P. \& MALIK, M. R. 1985 Stability experiments in the flow over a rotating disk. AIAA J. 23, 588-595. 